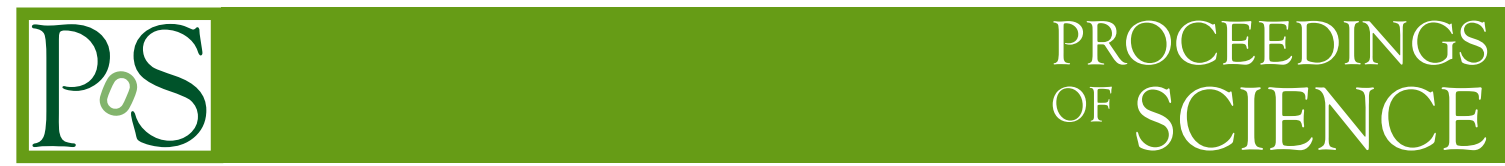

\title{
Summary of the latest PHENIX Heavy lon Results
}

\section{Christopher Pinkenburg* for the PHENIX Collaboration}

Brookhaven National Laboratory

E-mail: pinkenburg@bnl.gov

The PHENIX Experiment at the Relativistic Heavy Ion Collider of the Brookhaven National Laboratory has been taking data for over 10 years. The accumulated data sets span multiple beam energies and collision systems that provide an increasingly detailed picture of the hot and dense medium produced in central heavy ion collisions. This is a summary of our latest results for direct photons, cold nuclear matter effects and a beam energy scan.

The 2011 Europhysics Conference on High Energy Physics, EPS-HEP 2011,

July 21-27, 2011

Grenoble, Rhône-Alpes, France

${ }^{*}$ Speaker. 


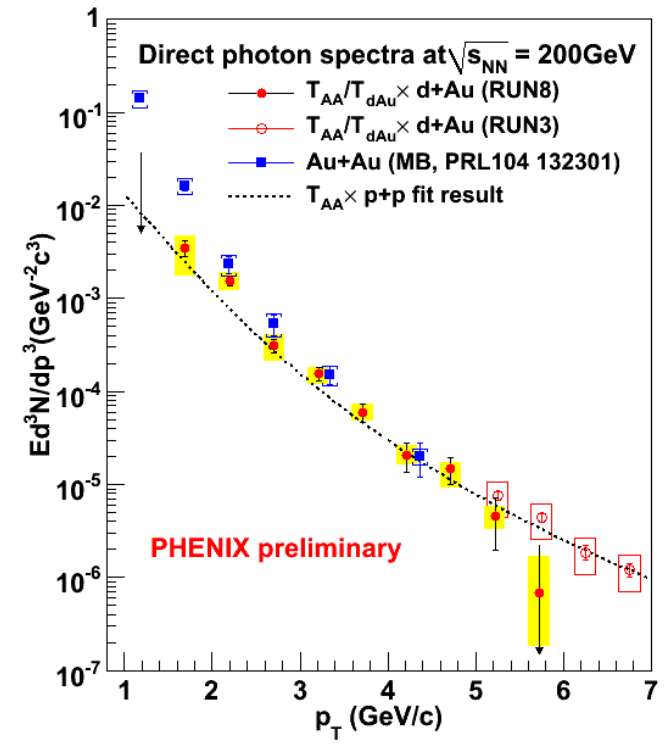

Figure 1: Invariant yield of direct photons as function of $p_{T}$. The red (blue) symbols show the invariant yields for $d+\mathrm{Au}(\mathrm{Au}+\mathrm{Au})$ collisions, the dashed line shows the scaled $p+p$ fit curve. While the $d+$ Au data agree with the scaled $p+p$ data, the $\mathrm{Au}+\mathrm{Au}$ data show an excess at low $p_{T}$.

\section{Direct photon $v_{2}$}

Direct photons are produced during the entire space-time history of relativistic heavy ion collisions $[1,2]$. Due to their small coupling they leave the collision region mostly without further interaction which makes them a direct probe of all stages of the collision. The transverse momentum ranges populated by various production mechanisms overlap, but event by event azimuthal asymmetries can provide additional information and the means to distinguish between sources of direct photons $[3,4]$. At higher $p_{T}\left(p_{T}>4 \mathrm{GeV} / \mathrm{c}\right)$ four sources of direct photons are predicted, each with a characteristic signature in $v_{2}$. Photons from initial hard scattering are expected to be emitted isotropically $\left(v_{2}=0\right)$. Fragmentation photons should have $v_{2}>0$ since the energy loss of the originating parton is smaller in the reaction plane. Bremsstrahlung and jet-conversion photons should have a negative $v_{2}$ because the average path length is larger perpendicular to the reaction plane. At low $p_{T}\left(1<p_{T}<4 \mathrm{GeV} / \mathrm{c}\right)$ thermal photons dominate the picture. They were first mea-

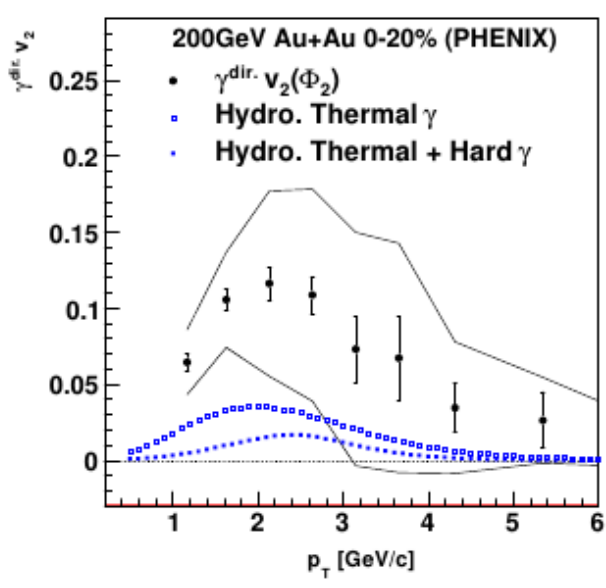

Figure 2: Azimuthal asymmetry $\left(v_{2}\right)$ of direct photons as a function of $p_{T}$ in the $20 \%$ most central $\mathrm{Au}+\mathrm{Au}$ collisions at $\sqrt{s_{N N}}=200 \mathrm{GeV}[6]$. The error bars indicate the statistical uncertainty, the lines the systematic uncertainty. The data are compared to a hydrodynamic calculation [7]. The open symbols include only thermal photons, the closed symbols take also prompt photons into account which is a more realistic scenario 


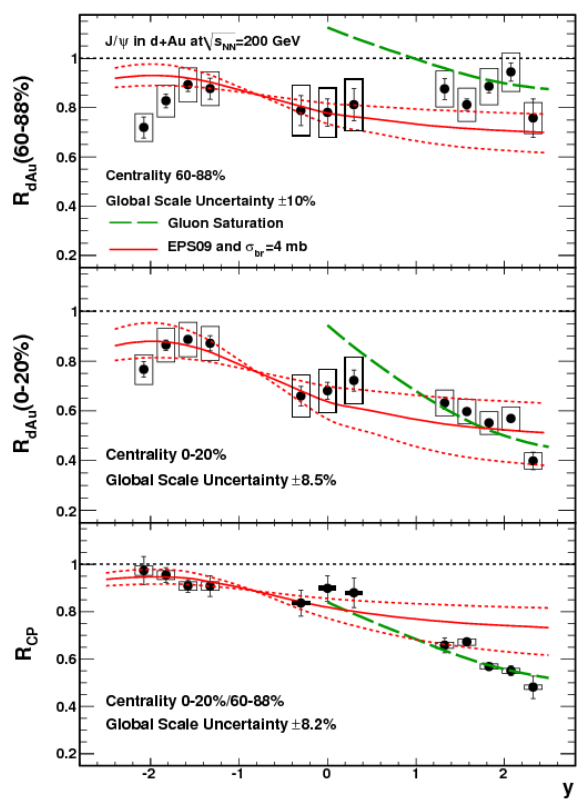

Figure 3: The nuclear modification factors $R_{d A u}$ for peripheral (top) and central (middle) events, and $R_{C P}$ as function of rapidity. The red line indicates a calculation using the EPS09 nuclear modified PDF set [10], the red dashed lines show the differences within the EPS09 nPDFs for a single parameter change that gives the largest variation. The green dashed line shows a calculation incorporating gluon saturation at small- $x[12,13]$

sured in [5] which showed a substantial excess in this region relative to the scaling of $p+p$ yields (Fig. 1). Using this measured excess it is now possible to extract the azimuthal asymmetry $v_{2}$ of direct photons from the inclusive photon $v_{2}$ which is dominated by decay photons. Fig. 2 shows the measurement of the direct photon $v_{2}$ as a function of $p_{T}$ for the $20 \%$ most central collisions at $\sqrt{s_{N N}}=200 \mathrm{GeV}$ [6]. The magnitude of $v_{2}$ at $p_{T} \approx 2 \mathrm{GeV} / \mathrm{c}$ is comparable to the elliptic flow measured for hadrons [8]. At high $p_{T}$ where isotropically emitted prompt photons dominate, $v_{2}$ approaches zero as expected. A comparison to a hydrodynamic calculation [7] shows that the measurement lies significantly above the model calculation. The discrepancy is even larger for a more realistic case in which prompt photons are included. This is a challenge for theory calculations and it is clear that the $v_{2}$ data for direct photons provide strong additional constraints on models.

\section{Cold Nuclear Matter (CNM) effects}

Imperative for the interpretation of heavy ion results is the measurement of cold nuclear matter effects in $d(p)+A$ collisions. $J / \Psi$ s offer a unique probe of cold nuclear matter effects in heavy ion collisions. As gluon-gluon fusion is the dominant $J / \Psi$ production process at RHIC, they can offer insight into gluon shadowing and anti-shadowing as well as gluon saturation at low $-x$. Given that we can separate events by impact parameter we can gather information on the geometric dependence of the nuclear modification. The PHENIX experiment measures $J / \Psi$ s through 2 decay channels: di-electrons at midrapidity and di-muons at forward/backward rapidities. Fig. 3 shows the nuclear modification factor $R_{d A u}$ as function of rapidity for central (top) and peripheral (middle) events as well as the ratio $R_{C P}$ between central and peripheral events (bottom) [9]. The quantity $R_{C P}$ has the advantage that many systematic uncertainties cancel in the ratio. These are compared to the EPS09 nuclear PDF set [10] using a breakup crossection of $\sigma_{b r}=4 m b$, chosen to match the unbiased backward rapidity $R_{d A u}$ data (red line) [11]. One sees a reasonable agreement at backward and midrapidities but a significant deviation in $R_{C P}$ at forward rapidities which hints at a centrality 


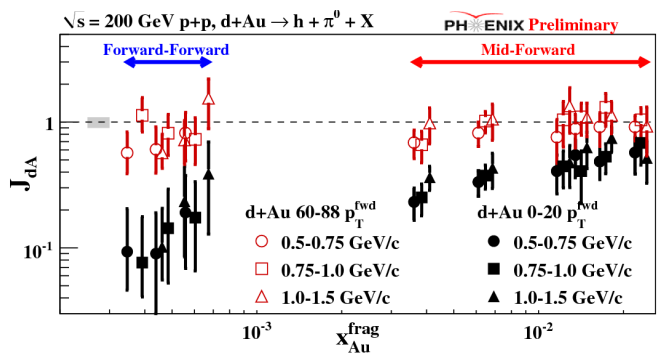

Figure 4: $J_{d A}$, the ratio of di-hadron production in $d+A u$ collisions to the binary scaled $p+p$ result versus $x_{A u}^{f r a g}$ a measure for the $x_{B j}$ of the parton in the $\mathrm{Au}$ nucleus for peripheral (60-88\%) and central (0$20 \%$ ) events. The vertical bars indicate the statistical uncertainty

dependence of nuclear effects. A calculation which incorporates gluon saturation effects at small- $x$ (green dashed line) $[12,13]$ is able to reproduce the suppression and its centrality dependence at forward rapidities but its predicted small enhancement at midrapidity due to double gluon exchange processes is not seen.

Low $-x$ gluon saturation has been predicted to be most apparent in di-hadron correlations in $d(p)+A$ collisions at forward (Au-going) rapidity where $x$ can be reasonably constraint $[14,15]$. PHENIX has recently measured di-hadron correlations of neutral pions in this kinematic region [16]. Fig. 4 shows the ratio of di-hadron production in $d+A u$ collisions to the binary-scaled $p+p$ result $\left(J_{d A}\right)$ as a function of $x_{A u}^{f r a g}$ a measure of the $x_{B j}$ of the parton in the Au nucleus. While there is no suppression for peripheral events we observe a significant suppression in central events which increases when probing more forward rapidities.

\section{Beam Energy Scan}

The large suppression of $\pi^{0}$ production which was first observed in $\sqrt{s_{N N}}=130 \mathrm{GeV} \mathrm{Au}+\mathrm{Au}$ collisions [17] is one of the most significant manifestations of the new state of matter produced in
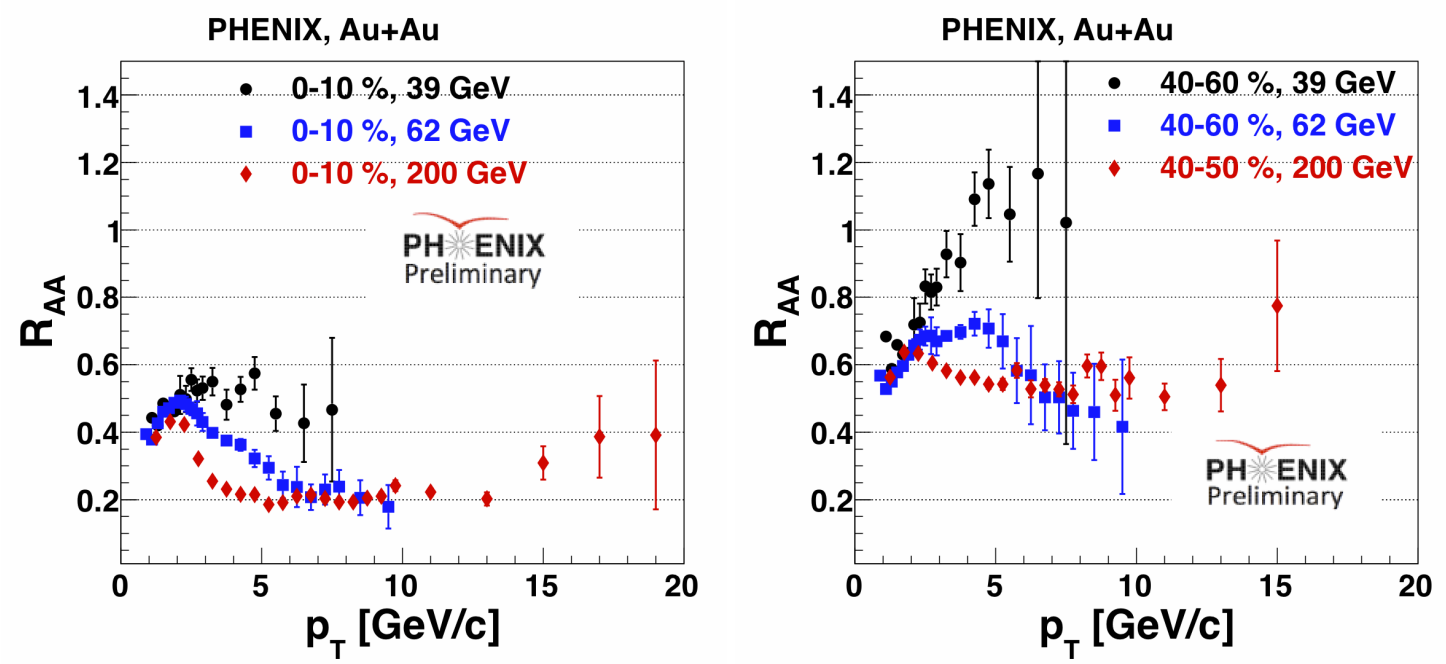

Figure 5: The nuclear modification factor $R_{A A}$ of $\pi^{0}$ production for central (left) and mid-peripheral (right) $\mathrm{Au}+\mathrm{Au}$ collisions as function of $p_{T}$ at $\sqrt{s_{N N}}=39,62.4$ and $200 \mathrm{GeV}$. The error bars represent the quadratic sum of the statistical and point to point systematic uncertainties. The necessary $p+p$ reference for $\sqrt{s_{N N}}=$ $39 \mathrm{GeV}$ was extrapolated from the E706 experiment at the Tevatron [18]. 

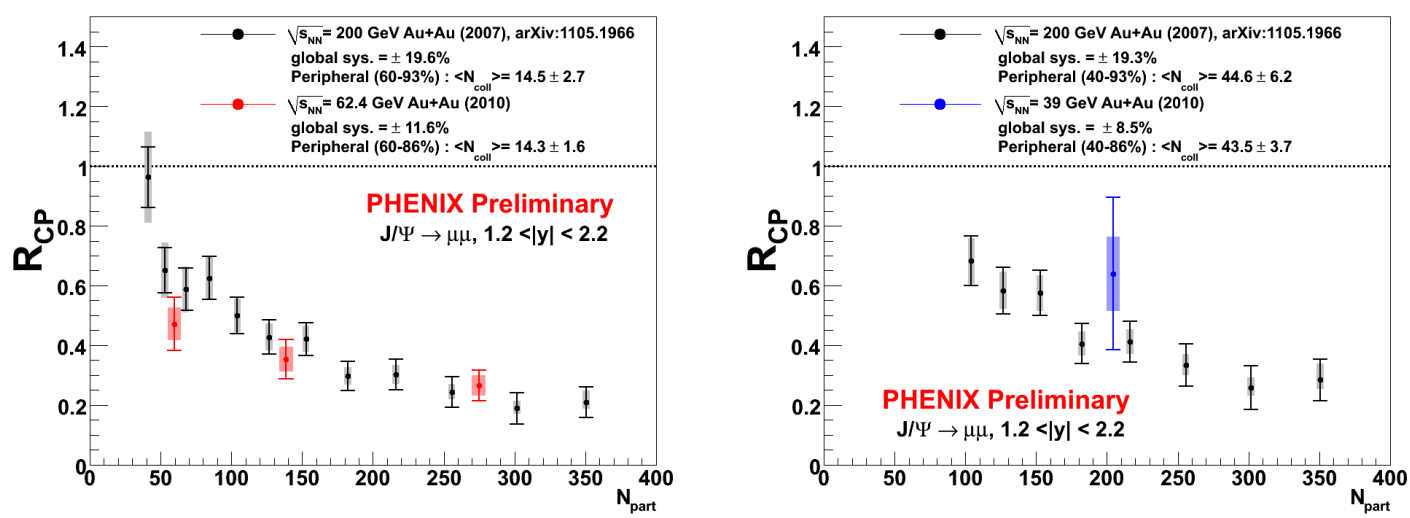

Figure 6: $R_{C P}$ for $J / \Psi$ production as function of the number of participants $\left(N_{\text {part }}\right)$ for $\sqrt{s_{N N}}=39,62.4$ and $200 \mathrm{GeV}$ in the rapidity range $1.2<|y|<2.2$.

ultra relativistic heavy ion collisions. One of the goals of the beam energy scan program is to search for the onset of this suppression. Fig. 5 shows the nuclear modification factor $R_{A A}$ for central and mid-peripheral collisions at $\sqrt{s_{N N}}=39,62.4$ and $200 \mathrm{GeV}$. All data sets exhibit a large suppression in central collisions suggesting that the new state of matter is produced even at $\sqrt{s_{N N}}=39 \mathrm{GeV}$. In mid-peripheral collisions a large suppression over the full $p_{T}$ range is only observed for $\sqrt{s_{N N}}=$ 62.4 and $200 \mathrm{GeV}$ while there is no suppression for the $\sqrt{s_{N N}}=39 \mathrm{GeV}$ data.

Quarkonia have long been proposed as a sensitive probe of the color screening length and deconfinement [19]. The modification by the hot dense medium will lead to a suppression of quarkonium production compared to $p+p$ collisions. Fig. 6 shows the $J / \Psi R_{C P}$ for $\sqrt{s_{N N}}=39$, 62.4 and $200 \mathrm{GeV}$. The suppression is of similar magnitude for all energies which is consistent with what was observed at SPS energies [20]. This picture gets complicated by the different magnitude of CNM effects $[9,21]$ which might account for a larger fraction of the total suppression at SPS energies compared to RHIC energies.

\section{Conclusion}

We have measured a significant azimuthal asymmetry $\left(v_{2}\right)$ for direct photons in the $1<p_{T}<$ $4 \mathrm{GeV} / \mathrm{c}$ range which is comparable to the $v_{2}$ observed for hadrons. It drops to zero at higher momenta where isotropically emitted prompt photons dominate. Its magnitude poses a serious challenge to theoretical calculations. We have measured sizable cold nuclear matter effects in forward $J / \Psi$ and di-hadron production which have to be taken into account when interpreting heavy ion results. The suppression of $\pi^{0}$ production in central events is similar for $\sqrt{s_{N N}}=39,62.4$ and $200 \mathrm{GeV}$ but we observe no suppression in mid-peripheral events at $\sqrt{s_{N N}}=39 \mathrm{GeV}$. For $J / \Psi$ production the suppression is of similar level for all three beam energies within the uncertainties.

\section{References}

[1] S. Turbide, R. Rapp, C. Gale, Phys. Rev. C 69, 014903 (2004) 
[2] W. Liu, R. J. Fries, Phys. Rev. C 77, 054902 (2008)

[3] S. Turbide, C. Gale, R. J. Fries, Phys. Rev. Lett. 96, 032303 (2006)

[4] R. Chatterjee, D. K. Srivastava, Phys. Rev. C 79, 021901 (2009)

[5] A. Adare et al. (PHENIX Collaboration), Phys. Rev. Lett. 104, 132301 (2010)

[6] A. Adare et al. (PHENIX Collaboration), arXiv:1105.4126v2 [nucl-ex]

[7] H. Holopainen, S. Rasanen, K. J. Eskola, arXiv:1104.5371 [hep-ph]

[8] S. S. Adler et al. (PHENIX Collaboration), Phys. Rev. Lett. 91, 182301 (2003)

[9] A. Adare et al. (PHENIX Collaboration), arXiv:1010.1246v1 [nucl-ex]

[10] J. Eskola, P. Paukkunen, C. A. Salgado, JHEP04, 065 (2009)

[11] R. Vogt, Phys. Rev. C 71, 054902 (2005)

[12] D. Karzeev, K. Tuchin, Nucl. Phys. A770, 40 (2006)

[13] D. Karzeev, K. Tuchin, Nucl. Phys. A735, 248 (2004)

[14] D. Karzeev, E. Levin, L. McLerran, Nucl. Phys. A748, 627 (2005)

[15] C. Marquet, Nucl. Phys. A796, 41 (2007)

[16] A. Adare et al. (PHENIX Collaboration), Phys. Rev. Lett. 107, 172301 (2011)

[17] K. Adcox et al. (PHENIX Collaboration), Phys. Rev. Lett. 88, 022301 (2002)

[18] L. Apanasevich et al. (E706 Collaboration), Phys. Rev. D 68, 052001 (2003)

[19] T. Matsui, H. Satz, Phys. Lett. B 178, 416 (1986)

[20] M. Abreu et al. (NA50 Collaboration), Phys. Lett. B 477, 28 (2000)

[21] R. Arnaldi (NA60), Nucl. Phys. A830, 345c (2009) 